\title{
Efectos del tratamiento de la dependencia a la heroína en la anomia, alienación y autoconcepto
}

\author{
Ramón Arce(1), Evaristo Díaz ${ }^{(2)}$, Ana Justo ${ }^{(1)}$ \\ (1) Departamento de Psicología Social y Básica, Universidad de Santiago de Compostela. \\ (2) ACLAD de A Coruña.
}

Enviar correspondencia a:

Ramón Arce. Departamento de Psicología Social y Básica. Facultad de Psicología. E-1782 Santiago de Compostela. Correo electrónico: psarce@usc.es

\section{RESUMEN}

En este estudio nos planteamos contrastar la efectividad de 3 programas de tratamiento de drogodependientes de heroína (Programa Libre de Drogas, Programa de Mantenimiento con Metadona y Programa de Mantenimiento con Naltrexona) en las medidas de la anomia, alienación y autoconcepto. Tomamos 91 pacientes a tratamiento por heroína: 31 a tratamiento con metadona, 30 a tratamiento con naltrexona y otros 31 en un programa libre de drogas. Los resultados mostraron que los pacientes estaban altamente alienados y no evidenciaron un efecto significativo del factor tratamiento. Los resultados también informan de niveles elevados de anomia entre los pacientes a tratamiento a la vez que un efecto significativo del factor tratamiento. En concreto, los pacientes a tratamiento con metadona perciben mayores niveles de anomia que aquellos a tratamiento con naltrexona. Asimismo, observamos que el autoconcepto era positivo para el conjunto de pacientes a la vez que encontramos que los pacientes a tratamiento con metadona presentaban un autoconcepto más bajo en algunos componentes que aquellos a tratamiento con naltrexona o en un programa libre de drogas. Finalmente, se discuten las implicaciones para los programas de intervención y prevención.

Palabras clave: drogodependencia, anomia, alienación, autoconcepto, metadona, naltrexona, programa libre de drogas.

\section{ABSTRACT}

The aim of this study was to contrast the effectiveness of three different treatment programmes for heroin abusers (drugfree treatment, antagonist treatment, and agonist treatment programmes) in mitigating the alienation, anomie and personal breakdown. 91 subjects were undergoing drug therapy: 30 with methadone treatment, 30 with naltrexone treatment and 31 with drug-free treatment. The results showed high alienation levels among the subjects in treatment and a non-significant effect was found in relation to the treatment factor. The results also indicate high levels of anomie among the subjects in treatment and a significant effect of the treatment factor i.e., higher anomie among patients treated with methadone $v$. naltrexone. Moreover, in the sample as a whole, self concept was positive at the same time we found that patients being treated with methadone had a lower self-concept in certain components than those being treated with naltrexone or taking part in a drug free programme. Implications for intervention and prevention programs are discussed.

Key words: Heroin abuse, anomie, alienation, self-concept, drug free treatment, antagonist treatment, agonist treatment.

\section{INTRODUCCIÓN}

$\mathbf{F}$ unes y Romaní (1985) consideran la drogodependencia como la interacción de tres factores: sustancia, contexto e individuo. Se entiende, de acuerdo con la OMS, por sustancia cualquier droga que, introducida en el organismo, puede modificar funciones de éste (v. gr., conducta, juicio, percepción o estado de ánimo"). La misma OMS ha clasificado las drogas según la peligrosidad. Las muy peligrosas son aquellas que crean dependencia física; que crean dependencia con más rapidez; y las que poseen mayor toxicidad. Los opiáceos y sus derivados serían, en este ranking de peligrosidad, las más peligrosas. Entre éstos se encuentra la heroína que, por su tasa y peculiaridades de consumo, se ha convertido en uno de los objetivos principales de tratamiento de nuestras Unidades de Atención a Drogodependientes (Becoña y Vázquez, 2001).

El contexto, segundo factor crítico en la adquisición de la drogodependencia, media la socialización del individuo. En un proceso de socialización "efectiva", el sujeto aprende los contenidos prosociales a la vez que a tener autocontrol, a aplazar la satisfacción 
de sus necesidades e impulsos, o a sentir culpa y remordimientos ante la transgresión de las normas sociales (Graña y García, 1994). No obstante, una socialización positiva puede desvanecerse posteriormente tal como bajo la contingencia de una adicción a las drogas. De hecho, los daños de una drogodependencia no son sólo físicos sino también sociales, psíquicos y cognitivos (i.e., Becoña, 1995). Esto es, los daños de la dependencia implican una desestructuración social y personal. La desestructuración personal y social se ha identificado sociológicamente con la alienación y anomia en tanto psicológicamente el reflejo central de la desestructuración es el autoconcepto (p.e., Lemert, 1967; Fernández-Ríos, 1994). La anomia, en principio y de acuerdo con las formulaciones de Durkheim, hace referencia a una propiedad del sistema social: la carencia de normas. Pero, como quiera que la adaptación a la sociedad se hace de un modo privado, individual y único, desde una óptica individual la anomia se ha operativizado como una falta de capacidad del individuo para adaptarse a las normas de conducta de su sociedad (Mclver, 1950; Riesman, 1981). En el caso de la inadaptación social, tal como es la drogodependencia y especialmente en el caso de los heroinómanos, la anomia es una forma de evasión a un ambiente asocial (Wells y Rankin, 1983) y una dificultad para la asunción de normas (Sancha y Puyó, 1997). La inadaptación social y personal trae consigo un distanciamiento de la sociedad. Esta distancia entre un individuo y los que le rodean (i.e., la sociedad), y con respecto a sí mismo (Fernández Ríos, 1994), se mide a través de la alienación. Son múltiples las repercusiones psicológicas para el sujeto de la anomia y alienación con implicaciones para la rehabilitación, reinserción y prevención de recaídas; en nuestro caso, la drogodependencia: pérdida de control personal, bajo cuidado autoprotectivo, reducción de conductas que promueven la salud, mayor número de episodios de enfermedad, peor autocalificación de la salud personal, apatía, resentimiento y carencia de integración social (p.e., Guthri y Tanco, 1980; Deflem, 1989; Fernández-Ríos, 1994).

El desarrollo ontogenético es el resultado de la socialización y éste se operativiza principalmente en el autoconcepto. El autoconcepto, la autoestima o el self, términos que pueden utilizarse indistintamente (Kolligian, 1990, pág. 273), entendidos como autoevaluación, constituyen las piedras angulares para el logro de un adecuado equilibrio psicológico y de un desarrollo personal satisfactorio, así como para la adquisición de conductas adaptativas y saludables (v. gr., Baron y Byrne, 1998). Complementariamente, una autoestima negativa con frecuencia es considerada como un factor de vulnerabilidad "genérica" que predispone al sujeto a un amplio espectro de desajustes comportamentales y afectivos. Se han planteado diversas hipótesis sobre la autoestima y la conducta desviada. Una primera hipótesis, sostenida por autores como Cohen (1955), Kaplan (1972) o Toch (1992), considera el comportamiento desviado como "mecanismo de compensación". Una segunda hipótesis, "hipótesis de protección", defendida tanto desde los modelos de la vulnerabilidad (p.e., Werner, 1986, Zubin, 1989) como desde los de competencia (v. gr., Wallston, 1992), sostiene que una alta autoestima "protege" al sujeto de las fuerzas que le incitan al desajuste o inadaptación social. Su planteamiento se enmarca dentro de las "teorías del control social" (Reckless, 196), que enfatizan el concepto de control para dar cuenta de por qué muchos individuos no desarrollan conductas antisociales. De acuerdo con esta hipótesis, para que los sujetos respeten una socialización positiva, habrán de existir mecanismos de contención o control capaces de contrarrestar las presiones internas (frustraciones, tensiones, necesidad de gratificación inmediata, etc.), y las externas (fracaso escolar, desempleo, discriminación, subculturas desviadas). Los métodos de control externo contemplados por Reckless son ejercidos por la familia y la comunidad (y por la sociedad en general), a través de procesos como el refuerzo positivo de las conductas socialmente deseables, o el castigo de las conductas antisociales, que inducirán al individuo a adoptar comportamientos socialmente adaptados. Los métodos de contención interna, según el autor, emanan del propio sujeto y son los más efectivos. Entre sus componentes figuran, como eje central, un autoconcepto sólido y positivo. Así, un autoconcepto positivo y robusto protegerá al sujeto de conductas antisociales como la drogodependencia, en tanto uno débil situará al individuo en una posición de vulnerabilidad para implicarse en grupos marginales y adoptar un estilo de vida antisocial. Una tercera hipótesis estaría propuesta por teorías del área de la criminología, que consideran un proceso inverso: "serían los actos delictivos (o más bien los mecanismos de sanción evocados por ellos) los que minarían la autoimagen. Las reacciones sociales (sanción, estigmatización, institucionalización) que se producen ante las conductas desviadas serían las que "incrustan" en la identidad del sujeto el rol de inadaptado y las que acaban mortificando un "yo" etiquetado (v. gr., Goffman, 1961; Becker, 1963; Lemert, 1967). En cualquier caso, se ha encontrado de un modo sistemático entre los drogodependientes, especialmente de la heroína, una baja autoestima (v. gr., Braucht y otros, 1973; Graña y García, 1994; Becoña y Vázquez, 2001).

Estas tres dimensiones, anomia, alienación y autoconcepto, merecen un acercamiento dual a la drogodependencia. Esta dualidad se refiere a que bien pueden ser la causa bien un efecto de la misma. No obstante, un tratamiento eficiente requiere de un control de éstas para garantizar una cierta probabilidad de rehabilitación y reinserción exitosa (Fernández-Ríos, 1994; Arce, Fariña y Novo, 2002). 
En relación con los procesos de socialización, internalización de los mismos y su relación con el tratamiento de la drogodependencia, nos hemos planteado un estudio cuyo objetivo principal fue la contrastación de posibles diferencias mediadas por el tratamiento de la dependencia a un opiáceo, la heroína, en la anomia, alienación y autoconcepto. Con este objetivo en mente nos planteamos contrastar tres hipótesis. Primera, se asume que los individuos dependientes de la heroína están más alienados y que el factor tratamiento desempeñará un rol significativo en el control o amortiguamiento de la alienación. En concreto, prevemos que los pacientes a tratamiento con metadona, por las características de los pacientes sometidos a este tratamiento y el tratamiento en sí, manifiesten menos acercamiento social que aquellos con otros tratamientos. Segunda, los sujetos a tratamiento por dependencia de la heroína percibirán el contexto social como anómico y la anomia será sensible al factor tratamiento en el que los sujetos a tratamiento con metadona percibirán un contexto social más anómico ya que este tratamiento no tiene por objeto potenciar la capacidad del paciente para adaptarse a las normas de conducta de su sociedad. Tercera, hipotetizamos, de acuerdo con las predicciones de Khaztzian (1999), los modelos de vulnerabilidad/competencia (Werner, 1986, Zubin, 1989; Wallston, 1992), y la "hipótesis de protección" (Reckless, 1961), un self negativo o en sus componentes entre los sujetos a tratamiento por dependencia de la heroína de lo que se derivaría una vulnerabilidad "genérica" que predispone al sujeto a un amplio espectro de desajustes que pueden conducirle a la recaída. Asimismo, sus componentes, tanto internos como externos, serán sensibles al factor tratamiento, ponderando el tratamiento con metadona más negativamente porque el tratamiento con metadona no se orienta a una mejora en el autoconcepto sino a la reducción de daños.

\section{MÉTODO}

\section{Muestra.}

La muestra se compone de 91 pacientes de la ACLAD (Asociación Ciudadana de Lucha y Ayuda a las Drogodependencias de A Coruña), tomados sobre la base de un muestreo teórico, de los cuales 31 estaban en tratamiento ambulatorio incluidos en un Programa Libre de Drogas, otros 30 eran pacientes ambulatorios incluidos en un Programa de Mantenimiento con Naltrexona y los 30 restantes están incluidos en un Programa de Mantenimiento con Metadona. Por edades, el total de la muestra tenía una edad media de $27.4\left(S_{x}=7.2\right)$ y una oscilación desde los 17 a los 44 años. Los sujetos del programa de tratamiento con metadona tenían un rango de edades desde los 21 a los 43 años, con una edad prome- dio de $31.4\left(S_{x}=5.8\right)$. Por su parte, los sujetos del programa de tratamiento con naltrexona oscilaban entre las edades de 19 y 43, con una media de 27.5 $\left(S_{x}=6.1\right)$. Los pacientes del programa de tratamiento libre de drogas abarcaban un rango de edades desde los 19 a los 44 años con una edad media de 31.3 $\left(S_{x}=6.02\right)$. Por géneros la muestra era mayoritariamente de varones, 68\% (70\% en los pacientes tratados con metadona; $86.7 \%$ en los tratados con naltrexona; y $77.4 \%$ en los del programa libre de drogas). Todos los sujetos contaban con una estructura familiar.

\section{Procedimiento y diseño.}

A todos los sujetos se les pasó una batería de cuestionarios a modo individual en los departamentos de la ACLAD. La batería incluía los siguientes instrumentos: la escala anomia de Srole (1956); la escala de alienación de Streuning y Richardson (1965); y el test del autoconcepto (Tennessee self) de Fitts (1964, 1965). Además, se recabaron datos de los archivos de la ACLAD de tipo demográfico (estado civil y estructura familiar) y de consumo (antecedentes familiares de drogadicción, antecedentes personales, tiempo de consumo continuado de heroína, cantidad y frecuencia del consumo, edad de comienzo de la dependencia). La participación fue voluntaria y no recibieron gratificación alguna por participar en el trabajo.

Los objetivos y clasificación de los pacientes según el programa administrado se describen a continuación. Los objetivos del Programa Libre de Drogas (PLD) son la extinción de la conducta drogodependiente, así como prevenir el uso de drogas en el medio social, restablecer el nivel de salud del paciente y resolver o reducir en la medida de lo posible conflictos personales, familiares y relacionales. El proceso de un programa con estas características se inicia con una desintoxicación, sea ésta hospitalaria o ambulatoria; sigue con un proceso de deshabituación para progresivamente llegar a una reinserción familiar, laboral y social libre ya de la adicción a las drogas, y con ello, transcurridos unos años en situación de abstinencia, se puede hablar de recuperación.

El Programa de Mantenimiento con Naltrexona (PMN) está basado en la ingesta de clorhidrato de Naltrexona, un antagonista opiáceo. La Naltrexona está indicada primordialmente en pacientes con fracasos previos en PLD, con una corta historia de consumo, con el suficiente apoyo social y familiar, con una aceptable adaptación social y una alta motivación para el abandono del consumo de drogas. El uso supervisado de esta sustancia actúa como un factor de protección para el paciente y su familia, ya que su finalidad es mantener al paciente ligado al tratamiento, así como evitar el consumo de opiáceos, modificar hábitos perjudiciales y restablecer su nivel de salud. 
El Programa de Mantenimiento con Metadona (PMM) tiene como objetivos disminuir el consumo ilegal de opiáceos, el uso o abuso de fármacos, reducir hábitos de riesgo y mejorar la calidad de vida, favorecer la captación y retención del paciente, así como disminuir la conducta antisocial y promover un cambio actitudinal y conductual. La metadona se utiliza como sustitutivo de la heroína ya que, por sus características, puede ser eficaz en cierto tipo de pacientes. Así, resulta indicado en personas que han fracasado en tratamientos previos, cuando existe una patología orgánica grave asociada, o en pacientes que, por llevar muchos años consumiendo o por otros motivos, no se plantean la desintoxicación. Como ventajas se apuntan la reducción de los problemas provocados por la situación ilegal de la heroína (adulteración de la sustancia, problemas con la autoridad, etc.); la reducción en la probabilidad de contraer ciertas enfermedades; disminución de la morbilidad; y facilitación de un mayor control social.. Por el contrario, se señala como el mayor problema de este programa de tratamiento la sustitución de un opiáceo por otro.

\section{Instrumentos de medida}

La "Escala de Anomia" de Srole (1956), que se compone de 9 ítems, mide el grado de integración del sujeto respeto a los demás y sociedad en general en oposición al sentimiento de identificación respecto a los demás. La unidimensionalidad de esta escala se evaluó mediante procedimientos de análisis estructural latente comprobándose la misma. Con nuestros sujetos, encontramos que la escala era consistente internamente, alpha de Chronbach $=.69$.

La "Escala de Alienación" de Streuning y Richardson (1965) se conformó en torno a los conceptos y teorías de Durkheim, Marx, May, Merton y Camus, y resultó de varios estudios factoriales de la misma a partir de unos trescientos enunciados originales. En nuestro estudio, la escala se mostró fiable, alpha de Chronbach de .83 .

Para evaluar el nivel de autoestima de nuestros sujetos experimentales tomamos el Test del autoconcepto o Tennessee Self (Tennessee Self-Concept) de Fitts (1965), que recoge y combina información de los aspectos internos y externos que conforman el self. Dentro de los aspectos internos del self se encuentran: la identidad; la autosatisfación; y el autocomportamiento. Por su parte, los aspectos externos son: el "sí mismo físico"; el "sí mismo moral-ético; el "sí mismo personal"; el "sí mismo familiar"; y el "sí mismo social." La combinación de los aspectos externos e internos nos da la puntuación total en test, que refleja el nivel global de la propia estima, concepto y comportamiento. Una puntuación total elevada indica que el sujeto tiende a autoestimarse, a considerarse persona meritoria y de valores, confianza en sí mismo y una actuación consecuente con esta confianza. Por contra, una puntuación total baja indica dudas en la valía personal y de la vivencia de uno mismo como persona no grata. Los sujetos con baja puntuación muestran ansiedad, depresión, infelicidad y poca confianza en sí mismos (Fitts, 1965). En nuestro estudio encontramos un apoyo a la consistencia interna de la escala tanto en la puntuación total, alpha de Chronbach .92, como en las subpuntuaciones que oscilaron entre .65 y .87 (véanse las puntuaciones por subcomponentes en la Tabla 1).

\section{Tabla 1. Estimación de la fiabilidad de los} subcomponentes del self.

\begin{tabular}{lc}
\hline Subomponente del self & Alpha de Chronba \\
\hline Autconcepto & .87 \\
Autosatisfacción & .83 \\
Autocomportamniento & .77 \\
Sí mismo físico & .75 \\
Sí mismo moral-ético & .65 \\
Sí mismo personal & .79 \\
Sí mismo familiar & .80 \\
Sí mismo social & .77 \\
\end{tabular}

\section{Análisis de datos.}

Dado el diseño y objetivos planteados, las herramientas estadísticas utilizadas se encaminaron a la obtención de diferencias de medias. De acuerdo con el diseño de cada caso, tomamos una herramienta u otra. Así, cuando comparamos la media de la población con un punto dado recurrimos a la "prueba t" para una muestra tomando como contraste un valor de prueba dado. Si, por el contrario, el diseño planteaba una comparación entre grupos, ejecutamos un ANOVA o un MANOVA según tuviéramos una o varias variables dependientes. En todas las contingencias se cumplían los presupuestos de estos análisis: la independencia de medidas; la homogeneidad de varianzas y la normalidad. Los contrastes se ejecutaron por el procedimiento de Sidak-Park, o sea, adoptamos una perspectiva conservadora ya que este procedimiento, basado en la prueba $t$, da lugar a límites más estrechos que el nivel de protección de Bonferroni.

\section{RESULTADOSY CONCLUSIONES}

\section{Alienación.}

Encontramos que los sujetos de nuestra muestra se encuentran alienados $(\underline{m}=63.24), t(90)=5.213$; 
$p<.001$ (ver nota al pie 1). Además, los resultados no muestran un efecto significativo terciado por el factor "tratamiento" (programa terapéutico: libre de drogas, naltrexona, y metadona), $\mathrm{F}(2,88)=2.913 ; \mathrm{MC}=490.690$; ns; T.E. $=.062$. Se cumple, de este modo, la previsión de la primera hipótesis en relación con que los pacientes a tratamiento por dependencia de la heroína se mostrarían alienados, pero no así la predicción en torno a un rol diferencial para los distintos tratamientos. En resumen, una de las implicaciones de la dependencia de opiáceos es la desestructuración personal, entendida aquí como distanciamiento emocional y desorientación, que, a tenor de nuestros datos, no se corrige simplemente con la administración de los tratamientos ordinarios previstos en el Plan Nacional de Drogodependencias. Sería, por tanto, preciso recurrir a programas complementarios en los que se diera cabida al tratamiento de la desestructuración personal para mejorar la salud mental y potenciar las probabilidades de rehabilitación y reinserción de estos pacientes.

\section{Anomia.}

Los resultados muestran que la población en tratamiento de drogodependencia de la heroína se encuentra en una situación anómica $(\underline{m}=5.1)$, entendida ésta como una medida de mala integración social, $\mathrm{t}(90)=2.999 ; \mathrm{p}<.01$ (ver nota al pie 2). Además, encontramos diferencias en anomia entre los tres grupos de tratamiento, $F(1,88)=3.374 ; \quad M C=12.724 ; \quad p<.05$; T.E. $=.071$. Los contrastes, procedimiento Sidak-Park, informan que los sujetos del grupo de tratamiento de mantenimiento con metadona $(m=5.3)$ están inmersos en un proceso más anómico $(p<.05)$ que los sujetos del grupo de naltrexona $(m=4)$. En suma, el tratamiento con metadona trae consigo o no llega a controlar las derivaciones anómicas, esto es, de aislamiento social de los sujetos en tratamiento por dependencia de heroína. Se cumplen así las predicciones de la segunda hipótesis tanto para los pacientes a tratamiento como para aquellos a tratamiento por metadona que perciben la sociedad aún más anómica. Las implicaciones negativas de la anomia, tanto para el individuo como para la sociedad, parecen claras desde la perspectiva de cualquiera de las teorías, tanto de las sociológicas como psicológicas revisadas en este trabajo (p.e., Durkheim, Merton, Maclver o Riesman). Un análisis puramente sociológico sostendría que la causa de esta anomia estaría en la sociedad con lo que sería necesaria una reestructuración social. Es evidente que éste puede ser uno de los motivos de origen de inicio de la drogodependencia. Una orientación más psicológico individualista apunta a la falta de capacidad del individuo para adaptarse a las normas como la causa de la anomia. En cualquier caso y desde una óptica más práctica, sería preciso un acercamiento preventivo al individuo a través de la implementación de programas comunitarios de tratamiento de la anomia sobre todo en los momentos vitales de riesgo de contraer una drogodependencia.

\section{Self.}

En general, el self ( $\underline{m}=302.7)$, entre los sujetos de la muestra a tratamiento por dependencia, es positivo, $\mathrm{t}(90)=12.985 ; \mathrm{p}<.001$ (ver nota al pie 3). Así, estos sujetos tienen presente una de las bases psicológicas implicadas en la adquisición y mantenimiento de un equilibrio psicológico y un desarrollo personal satisfactorio. No se cumple así, de acuerdo con las hipótesis de protección/vulnerabilidad/competencia, que estos sujetos sean más vulnerables por causa del self (Hipótesis 3). No obstante, el self está mediado en su conjunto por los niveles del factor "tratamiento" (programa terapéutico: libre de drogas, naltrexona y metadona), $F_{\text {multivariada }}(6,174)=2.496 ; p<.05 ;$ T.E. $=079$. Por su parte, los resultados univariados ponen de manifiesto efectos significativos en los tres componentes internos del self: la autosatisfacción, la identidad y el autocomportamiento (véase la Tabla 2).

Tabla 2. Pruebas de los efectos inter-sujetos

\begin{tabular}{lrrrrrrr}
\hline Variable & $\mathbf{M C}$ & $\mathbf{F}$ & $\mathbf{p}$ & $\mathbf{E t a}^{2}$ & $\mathbf{m}_{\mathbf{l d}}$ & $\mathbf{m}_{\mathbf{n}}$ & $\mathbf{m}_{\mathbf{m}}$ \\
\hline Identidad & 1210.653 & 5.839 & .004 & .117 & 110.3 & 114.3 & 101.9 \\
Autosatisfacción & 869.855 & 3.795 & .026 & .079 & 98.2 & 100.3 & 90.1 \\
Autocomportamiento & 185.925 & .989 & .376 & .022 & 97.5 & 100.3 & 95.3 \\
\hline
\end{tabular}

Nota: G.L. $(2,88) . m_{l d}=$ media grupo libre de drogas; $m_{n}=$ media grupo de naltrexona; $m_{m}=$ media de grupo de metadona

\footnotetext{
1 Ejecutamos una " $t$ " para una muestra siendo el valor de prueba de 56 que es punto de la escala que separa la alienación de la no alienación.

2Ejecutamos una "t" para una muestra siendo el valor de prueba de 4 que es punto de la escala que separa la anomia de la no anomia.

${ }^{3}$ Ejecutamos una " $t$ " para una muestra siendo el valor de prueba de 250 que es punto de la escala que separa la región de un self positivo del negativo.
} 
Los contrastes simples, procedimiento Sidak-Park, informan de una mayor identidad y autosatisfacción en el grupo de tratamiento con naltrexona en comparación con el de metadona $(p<.01)$.

En consecuencia, procedimos a llevar a cabo análisis para los subcomponentes externos implicados en la identidad y la autosatisfacción. Tal y como era de prever, constatamos la existencia de diferencias multivariadas en los subcomponentes de la identidad moduladas por el tratamiento, $F_{\text {multivariada }}$ $(10,170)=3.468 ; p<.001 ;$ T.E. $=.169$. Los efectos univariados, que pueden verse en la Tabla 3 , informan de diferencias en la identidad física, familiar y social.

A su vez, los contrastes simples, indican que los pacientes del programa de mantenimiento con metadona presentan una identidad física significativamente más baja que los pacientes del programa libre de drogas $(p<.001)$ y del programa de mantenimiento con naltrexona $(p<.01)$. En otras palabras, los pacientes a tratamiento con metadona tienen una percepción más negativa de sí mismos en términos de su estado de salud, aspecto físico, capacidad y sexualidad. En la identidad familiar $(p<.05)$ y social $(p<.01)$ observamos que, en comparación con el programa de mantenimiento con naltrexona, los pacientes del programa de tratamiento con metadona informan de una identidad familiar y social más baja. Esto es, las redes familiar y social, y, por extensión, el apoyo que éstas prestan es percibido como inferior en aquellos pacientes a tratamiento de metadona. Evidentemente, esta percepción implica que los sujetos ya no recurran a buscar apoyo en estas redes (véase Gracia, Herrero y Musitu, 1995).

En los subcomponentes externos de la autosatisfacción también encontramos efectos multivariados significativos mediados por el factor tratamiento, $F_{\text {mul- }}$ tivariada $(10,170)=1.881 ; p<.05 ;$ T.E. $=.100$. Los efectos univariados evidencian diferencias en las variables autosatisfacción física, personal y social (véase la Tabla 4).

Por su parte, los contrastes simples muestran diferencias entre los grupos de tratamiento a metodona y naltrexona en la autosatisfacción física $(p<.05)$; en la autosatisfacción personal $(p<.05)$ y autosatisfacción social $(p<.01)$. En todas estas comparaciones los sujetos del programa de tratamiento con metadona presentan una autosatisfacción más baja. Las derivaciones para la evaluación del tratamiento son claras: los pacientes tratados con metadona "sienten" que su estado de salud, aspecto físico, su capacidad, sexualidad, sus valores interiores, sentimiento de adecuación como persona, la valoración de su propia personalidad, y la valoración de la interacción con otras personas, es inferior a la de otros pacientes a tratamiento; en este caso, por naltrexona.

Tabla 3. Pruebas de los efectos inter-sujetos

\begin{tabular}{lrrrrrrr}
\hline Variable & MC & $\mathbf{F}$ & $\mathbf{p}$ & $\mathbf{E t a}^{\mathbf{2}}$ & $\mathbf{m}_{\mathbf{l d}}$ & $\mathbf{m}_{\mathbf{n}}$ & $\mathbf{m}_{\mathbf{m}}$ \\
\hline Identidad física & 121.277 & 10.403 & .000 & .191 & 23.2 & 22.6 & 19.5 \\
Identidad moral-ética & 1.82 & .141 & .868 & .003 & 21.4 & 21.8 & 21.4 \\
Identidad personal & 27.027 & 1.84 & .165 & .040 & 22.3 & 24 & 22.3 \\
Identidad familiar & 97.62 & 4.296 & .017 & .089 & 22.3 & 23.2 & 19.7 \\
Identidad social & 90.177 & 6.305 & .003 & .125 & 21 & 22.7 & 19.2 \\
\hline
\end{tabular}

Nota: G.L. $(2,88)$. $m_{l d}=$ media grupo libre de drogas; $m_{n}=$ media grupo de naltrexona; $m_{m}=$ media de grupo de metadona

Tabla 4. Pruebas de los efectos inter-sujetos

\begin{tabular}{lrrrrrrr}
\hline Variable & MC & $\mathbf{F}$ & $\mathbf{p}$ & $\mathbf{E t a}^{2}$ & $\mathbf{m}_{\mathbf{l d}}$ & $\mathbf{m}_{\mathbf{n}}$ & $\mathbf{m}_{\mathbf{m}}$ \\
\hline Autosatisfacción física & 66.385 & 3.506 & .034 & .074 & 20.8 & 21.2 & 18.4 \\
Autosatisfacción moral-ética & 23.752 & 1.467 & .236 & .032 & 20.4 & 18.8 & 19 \\
Autosatisfacción personal & 67.907 & 3.474 & .035 & .073 & 18.9 & 19.6 & 16.7 \\
Autosatisfacción familiar & 17.872 & .854 & .429 & .019 & 17.4 & 18 & 16.5 \\
Autosatisfacción social & 84.411 & 4.982 & .009 & .102 & 20.7 & 22.7 & 19.4 \\
\hline
\end{tabular}

Nota: G.L. $(2,88) \cdot m_{l d}=$ media grupo libre de drogas; $m_{n}=$ media grupo de naltrexona; $m_{m}=$ media de grupo de metadona 
En suma, en general los pacientes a tratamiento por dependencia de la heroína cuentan con un factor de protección a nivel del self. No en vano, el self informa que gozan de un equilibro psicológico y un desarrollo personal satisfactorio, lo que se traduce en una mayor probabilidad de éxito del tratamiento. No obstante, aquellos a tratamiento por metadona, con lo que recabamos apoyo a la segunda implicación de la hipótesis 3, conforman una población "más vulnerable" a desajustes comportamentales y afectivos, o "menos competente" para afrontar tales desajustes con lo que presentan, a priori, un factor de protección más bajo para la prevención de recaídas.

\section{DISCUSIÓN: IMPLICACIONES PARA LA EL TRATA- MIENTO}

Es obligado comenzar con una autocrítica del estudio aquí mostrado a fin de establecer el potencial exacto de las implicaciones a perfilar a partir de este estudio. Así, a priori, son dos las matizaciones que merecen nuestros resultados. Primera, éstos no pueden generalizarse más allá de los sujetos dependientes de heroína. Segundo, si bien los resultados van, generalmente, en línea con las predicciones de la literatura al respecto, con el fin de poder generalizarlos sería conveniente replicarlos en otras unidades de tratamiento. De hecho, éstos podrían estar "contaminados" por un efecto de la unidad asistencial en la que fueron obtenidos, entre otros.

Sobre esta base que nos debe llamar la atención sobre el alcance de las misma, hemos dibujado las siguientes implicaciones para los dos niveles de actuación, social y personal, que engloban la problemática en su conjunto:

a) A nivel social. La percepción de una anomia social, que contamina a los sujetos de nuestra muestra, trae consigo carencia de autonomía; pérdida del sentido psicológico de comunidad o desconfianza en los sistema comunitarios (Sue y Zane, 1980; Gordon, 1985; Fernández-Ríos, 1994) con lo que el tratamiento no ha resuelto totalmente el problema planteado y, por tanto, es preciso complementarlo para proceder a una prevención terciaria. En suma, es necesaria una intervención en el nivel social, tal como la promoción de programas comunitarios que promuevan una participación en la rehabilitación y reinserción, a la que no dan solución los tratamientos tradicionales.

b) A nivel personal. Los sujetos en tratamiento precisan de una atención personalizada para hacer frente a las repercusiones psicológicas de la alienación y anomia: pérdida de control personal, bajo cuidado autoprotectivo, reducción de conductas que promueven la salud, mayor número de episodios de enfermedad, peor autocalificación de la salud personal, apatía, resentimiento y carencia de integración social (p.e., Guthri y Tanco, 1980; Deflem, 1989; FernándezRíos, 1994). En lo referente al self, en principio nuestros sujetos cuentan con adecuado equilibrio psicológico que favorece la adquisición de conductas adaptativas y saludables, no presentado una predisposición a la vulnerabilidad. Ahora bien, el grupo de tratamiento con metadona precisa ser complementado en su tratamiento con un programa encaminado a fortalecer la identidad física, familiar y social a la vez que la autosatisfacción física, personal y social.

En conclusión, los diversos programas de tratamiento requieren ser complementados en algunos efectos que no controlan o generan por sí mismos. En este contexto, los tratamientos propuestos en la literatura para superar estos efectos no controlados por los tratamientos originales van desde la participación y la potenciación (Donovan y O'Leart, 1983; Orford, 1985; Fernández-Ríos, 1994), para superar los efectos de la anomia y alienación social, hasta los programas de pensamiento prosocial para subsanar las deficiencias o lagunas de orden cognitivo (véase López, Garrido y Ross, 2001). No obstante, previamente a cualquier intervención es conveniente realizar un pretratamiento de menor coste tanto social como personal. Nos referimos a enfrentar a los sujetos a un posible déficit metacognitivo (Perkins, 1989) que diera cuenta de un afrontamiento directo del problema.

\section{REFERENCIAS}

Arce, R., Fariña, F., y Novo, M. (2002). Evaluación de menores en proceso de tratamiento por comportamiento antisocial. En F. Fariña, y R. Arce (Eds.), Avances en torno al comportamiento antisocial, evaluación y tratamiento. Madrid: Ministerio de Trabajo y Asuntos Sociales.

Baron, R. A., y Byrne, D. (1998). Psicología social (8 ed.). Madrid: Prentice Hall.

Becker, H. (1963). Outsiders. Nueva York: Free Press.

Becoña, E. (1995). Drogodependencias. En A. Belloch, y B. Sandín (Eds.), Manual de Psicopatología (Vol. I). Madrid: Mcgraw-Hill.

Becoña, E., y Vázquez, F. L. (2001). Heroína, cocaína y drogas de síntesis. Madrid: Síntesis.

Braucht, G.N., Brakarsh,D., Follingstad,D., y Berry, K.L. (1973). Deviant drug use in adolescence: a review of psychosocial correlates, Psychological Bulletin, 79, 92-106.

Cadafalch, J. y Casas, M. (1993). El paciente heroinómano en el hospital general. Bilbao: Plan Nacional sobre Drogas.

Cohen, A. (1955). Delinquent boys. Nueva York: Free Press. 
Deflem, M. (1989). From anomie to anomia and anomic depression: a sociological critique on the use of anomie in psychiatric research. Social Science and Medicine, 29, 627-634.

Donovan, D. M. y O'Leart, M. R. (1983). Control orientation drinking behaviour and alcoholism. En H. M. Lefcourt (Ed.), Research with the locus of control construct, vol. 2: Development and social problems. Nueva York: Academic Press.

Fernández-Ríos, L. (1994). Manual de psicología preventiva. Teoría y práctica. Madrid: Siglo XXI.

Fitts, W. (1965). Manual: Tennessee self scale. Nashville, Tenn.: Counselor Recordings and Tests.

Funes, J., y Romaní, O. (1985). Dejar la heroína. Madrid: Ministerio de Trabajo y Seguridad Social.

Goffman, E. (1961). Asylums: essays on the social situation of mental patients and other inmates. Garden City, N.Y.: Anchor Books.

Gordon, A. (1985). Learned helplessness and community development: a case study. Journal of Community Psychology, 13, 327-337.

Gracia, E., Herrero, J., y Musitu, G. (1995). El apoyo social. Barcelona: PPU.

Graña, J. L. (1994). Conductas adictivas. Teoría, evaluación y tratamiento. Madrid: Debate.

Guthri, G. M., y Tanco, P. P. (1980). Alienation. En H. C. Triandis, y J. G. Draguns (Comps.), Handbook of crosscultural psychology (Vol. 6). Psycopathology. Boston, Mass.: Allyn and Bacon.

Kaplan H. B. (1972). Toward a general theory of psychosocial deviance: the case of aggressive behavior. Social Science and Medicine, 6, 539-617.

Khantzian, E. J. (1999). Treating addiction as a human process. New Jersey: Jason Aronson.

Kolligian, J. 1(990). Perceived fraudulence as a dimension of perceived incompetence. En R. J. Sternberg, y J. Kolligian (Comps.), Competence considered. New Haven: Yale University Press.

Lemert, E. M. (1967). Human deviance, social problems and social control. Nueva York: Prentice- Hall.

López, Ma. J., Garrido, V., y Ross, R. R. (2001). El programa del pensamiento prosocial: avances recientes. Valencia: Tirant lo Blanch.
Mclver, R. M. (1950). The ramparts we guard. Nueva York: The Macmillan Company.

Orford, J. (1985). Excessive appetites: a psychological view of addictions. Nueva York: John Wiley and sons.

Perkins, D. N. (1989). Reasoning as it and could be: an empirical perspective. En D.M. Topping, D. C. Crowell, y V. N. Kobayaski (Eds.), Thinking across cultures: the third international conference on thinking. Hillsdale, N.J.: LEA.

Reckless, W. C. (1961). A new theory of delinquency and crime. Federal Probation, 25, 42-46.

Riesman, D. (1981). La muchedumbre solitaria. Barcelona: Paidós.

Sancha, V., y Puyó, M. C. (1997). Nuevas tendencias en la intervención con jóvenes infractores. En J. Urra y M. Clemente (Coords.), Psicología jurídica del menor (pp. 401-427). Madrid: Fundación Universidad Empresa.

Srole, L. (1956). Social integration and certain corolaries. American Sociological Review, 21, 709-716.

Streuning, E. L., y Richardson, A. H. (1965). A factor analytic exploration of the alienation, anomie, and authorianism domain. American Sociological Review, 30, 768-776.

Sue, S., y Zane, N. (1980). Learned helplessness theory and community psychology. En M. S. Gibbs, J. R. Lachenmeyer, y J. Sigel (Comps.), Community psychology: theoretical and empirical approaches. Nueva York: Gardner Press.

Toch, H. (1992). Violent men. An inquiry into the psychology of violence. Washington: American Psychological Association.

Wallston, K. A. (1992). Hocus-pocus, the focus isn't strictly on locus: Rotter's social learning theory modified for health. Cognitive Therapy and Research, 16, 183-199.

Wells, L. E., y Rankin, J. H. (1983). Self-concept as a mediating factor in delinquency. Social Psychology Quarterly, 46, 11-22.

Werner, E. E. (1986). The concept of risk from a developmental perspective. En B. K. Keogh (Comp.), Advances in special education (vol. 5). Greenwich: Jai Press.

Zubin, J. (1989). Suiting therapeutic intervention to the scientific models of aetiology. British Journal of Psychiatry, 115(5), 9-14. 